\title{
Motor Channelling for Safe and Effective Dynamic Constraints in Minimally Invasive Surgery
}

\author{
Maria Grammatikopoulou, Konrad Leibrandt, and Guang-Zhong Yang, Fellow, IEEE
}

\begin{abstract}
Motor channelling is a concept to provide navigation and sensory feedback to operators in master-slave surgical setups. It is beneficial since the introduction of robotic surgery creates a physical separation between the surgeon and patient anatomy. Active Constraints/Virtual Fixtures are proposed which integrate Guidance and Forbidden Region Constraints into a unified control framework. The developed approach provides guidance and safe manipulation to improve precision and reduce the risk of inadvertent tissue damage. Online three-degree-of-freedom motion prediction and compensation of the target anatomy is performed to complement the master constraints. The presented Active Constraints concept is applied to two clinical scenarios; surface scanning for in situ medical imaging and vessel manipulation in cardiac surgery. The proposed motor channelling control strategy is implemented on the da Vinci Surgical System using the da Vinci Research Kit (dVRK) and its effectiveness is demonstrated through a detailed user study.
\end{abstract}

\section{INTRODUCTION}

Minimally Invasive Surgery (MIS) has become the gold standard in a number of surgical procedures. The main reasons for this development are the reduced time of recovery, blood loss and trauma in MIS in comparison to open surgery. The introduction of robotic systems has shown advantages compared to traditional MIS, such as increased precision and safety. Despite technological advances, teleoperated instruments provide reduced haptic perception in comparison to traditional laparoscopic tools. This lack of force sensing is a drawback of surgical robots and is essential in many surgical tasks. Surgeons perceive limited haptic cues of forces exerted on the tissue only through deformation observed in the stereoscopic video.

Therefore, an essential consideration is to incorporate haptic feedback into the human-machine interface enabling the operator to perform more precise and safer procedures. A concept developed to this end is Active Constraints (ACs), also known as Virtual Fixtures (VFs). Active Constraints are control algorithms which generate force feedback from virtual environments. Guidance Active Constraints (GACs) assist tool navigation along predefined paths. This path can be generated from pre- or intra-operative data through imaging techniques or from Learning from Demonstration (LfD) algorithms. GACs have been previously proposed for cardiac surgery [1], [2] and for bimanual coordination of surgical

All authors are with the Hamlyn Centre for Robotic Surgery, Imperial College London, SW7 2AZ, United Kingdom. (email: m.grammatikopouloul4@imperial.ac.uk). Maria Grammatikopoulou was supported by an Institute of Global Health Innovation scholarship. Konrad Leibrandt was supported by the President's PhD Scholarship of Imperial College London and EPSRC UK. tasks, such as suturing [3]. In contrast, Forbidden Region Active Constraints (FRACs) restrict the tool from operating in areas where tissue manipulation can cause unintentional damage. FRACs have been proposed for orthopedic procedures [4], cardiac surgery [5] and Ear, Nose and Throat (ENT) operations [6]. One fundamental difference between these two types of constraints is that classical GACs interfere continuously during tool manipulation while FRACs act on demand when constraint penetration is detected. Previous research suggests control methods for either GACs or FRACs but not a unified control strategy to achieve both goals simultaneously due to the contradicting force profiles and goals of these types of constraints.

Another challenge of Active Constraints frameworks is the introduction of instabilities to the robotic control system. Switching between control strategies result in altering among a number of closed-loop systems leading to oscillations. Rendering forces may lead to an increase of kinetic energy resulting to unstable and undesired autonomous behaviour. Therefore, a system which dissipates energy is an safer alternative to achieve bounded rendering forces [7].

Furthermore, ACs have been exploited to account for dynamically changing anatomy. Implementations include master-slave platforms [8], hands-on manipulators [9] and flexible robots [10]. Pre-operative offline motion planning is often required to compensate anatomy dynamics [11]. For accurate motion compensation, it is necessary to take into account system and online sensing delays. In [12] onedimensional online motion prediction and compensation is employed to achieve this goal using Fast-Fourier Transform (FFT) in conjunction with an Extended Kalman Filter (EKF).

The contributions of this work include: I) Unifying Guidance and Forbidden Region Constraints to facilitate precision and safety simultaneously while avoiding controller switching and achieving smooth and continuous force-torque rendering, II) providing six-degree-of-freedom (DoF) constraints for the master position and orientation for different operational scenarios, III) combining a control strategy with the proposed force-torque profile for additional properties, in this case motion redirection and kinetic energy dissipation and IV) performing 3-DoF real-time compensation of the dynamic surgical environment that leads to a semi-static operator frame of reference.

The main clinical applications of the proposed design are surface scanning for in situ medical imaging and microvascular anastomosis in Totally Endoscopic Robotic Coronary Artery Bypass (TECAB). In surface scanning, the acquired images are more informative and consistent when defor- 
mation is minimized and tool orientation is following the surface normal. In microvascular anastomosis, the motion of the anatomy has to be followed while minimum tension must be applied to the vessels during manipulation.

The proposed motor chanelling control scheme is implemented on the da Vinci Surgical System (Intuitive Surgical Inc., CA) (Fig. 4) using the da Vinci Research Kit (dVRK) [13]. Its effectiveness is demonstrated by the conducted user studies simulating surface scanning for in situ medical imaging and vessel manipulation in cardiac surgery.

\section{Master Manipulator Active Constraints CONTROLLER}

\section{A. Constraint Generation and Evaluation}

The target anatomy is represented as a set of threedimensional (3D) points and their corresponding normal vectors. The point cloud can be derived either from preoperative scanning of the anatomy, intra-operative imaging and computer vision techniques or from LfD through which a desired path for haptic guidance is obtained. However, the constraint generation process is not in the scope of this work. In our experimental implementation, the 3D surface of the phantom was obtained through CT scanning. The derived virtual model is registered with the master-slave workspaces. The constraints are evaluated by calculating the shortest distance between the tool end effector and the constraint surface in the master workspace. This computation is performed in real-time by organizing the points in a k$\mathrm{d}$-Tree on which a k-nearest neighbour search is performed. Once the closest point is identified, it is investigated whether the tool has penetrated the constraint boundary.

\section{B. Unified Guidance and Forbidden Region Force-Torque Profile}

Safe and effective constraints are realized by defining a force-torque profile that combines guidance and forbidden region constraints in a single control law. The motivations of integrating guidance and forbidden region constraints in a single force-torque profile are: I) to simultaneously regulate motion around the surface to improve precision, while minimizing penetration of the anatomy by the tool to increase safety, II) to respect the user's intentions during tool manipulation through selective force rendering and III) to avoid switching between attractive and repulsive forces thus preventing discontinuities. The model is utilized to regulate both the position and orientation of the tool and the master input. The proposed nonlinear profile (Fig. 1 - red) which combines the desired control objectives is described by:

$$
\begin{aligned}
f_{\text {scale }} & =\left\{\begin{array}{lll}
0 & \text { if } & d \geq d_{\max } \\
f_{A} & \text { if } & d_{\max }>d>-d_{\text {high }} \\
1 & \text { if } & d \leq-d_{\text {high }}
\end{array}\right. \\
f_{A} & =k_{0}\left\|\mathbf{p}_{\mathbf{t}}\right\|^{2}\left(\left\|\mathbf{p}_{\mathbf{t}}\right\|-d_{\max }\right)^{2} e^{k_{1}\left\|\mathbf{p}_{\mathbf{t}}\right\|}
\end{aligned}
$$

with (2) as in [14], $k_{0}$ a scalar constant, $d_{\max }$ the distance from the anatomy at which the constraint force-torque is activated, $d_{\text {high }}$ the distance at which the force-torque is

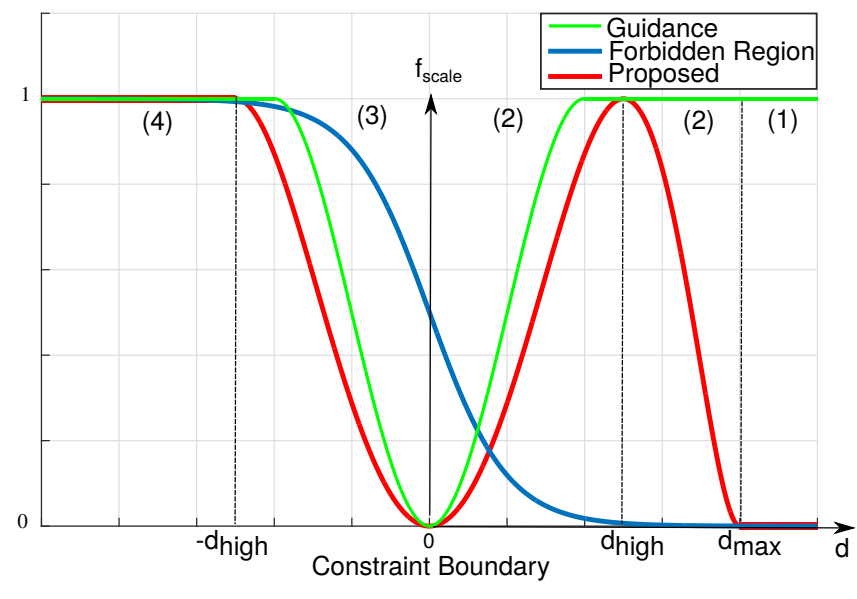

Fig. 1. Guidance (green), forbidden region (blue) and proposed (red) forcetorque profiles with respect to signed distance

maximum, $\mathbf{p}_{\mathbf{t}}=\mathbf{p}-\mathbf{p}_{\mathbf{d}}$ the penetration vector and $\mathbf{p}, \mathbf{p}_{\mathbf{d}}$ the current and desired end effector positions (Fig. 2).

The classical guidance and forbidden region force profiles are depicted in Fig. 1. The guidance profile (green) indicates that a force-torque is always applied to the user during tool manipulation. This force-torque is decreasing around the constraint boundary and becomes zero only when the target is reached, thus the operator loses authority to some extent. In contrast, the forbidden region force profile (blue) does not interfere with the user unless constraint penetration is detected, hence its main objective is to increase safety. The asymptotic behaviour of the proposed profile follows the classical forbidden region profile (blue) (regions 1 and 4) while it behaves similar to a guidance force-torque profile locally at the constraint boundary (regions 2 and 3).

The master workspace is divided in four control zones as depicted in Fig. 2. In the following, the signed distance $d$ denotes the distance of the tool tip with respect to the boundary stating if penetration has occurred. A positive sign implies that the tool is outside of the anatomy while a negative sign indicates constraint penetration. The four regions are described as follows:

1) The operator is allowed to move the instrument tip freely without any guidance force-torque applied towards the anatomy. This accounts for the fact that the desired tool pose is not known and the operator has unrestrictive control of the instrument.

2) When the end effector is within the transition zone $d_{\max }$, an attractive force is applied to assist the user in establishing contact with the anatomy. Simultaneously, a rotational torque starts to increase aligning the tool with the desired orientation. Their maximum values are rendered at distance $d_{\text {high }}$ from the surface.

3) In case of constraint violation, a retracting force is applied to redirect the instrument tip to the surface of the anatomy reaching its maximum amplitude at $-d_{\text {high }}$.

4) The maximum retraction force is maintained below $-d_{\text {high }}$ to redirect the user towards the surface preventing tissue damage. 


\section{Active Constraints in Tool Orientation}

Another control objective is to align the tool tip with a desired orientation for the performed task. Let $R=\left[\begin{array}{lll}\mathbf{t}_{\mathbf{x}} & \mathbf{t}_{\mathbf{y}} & \mathbf{n}\end{array}\right]$

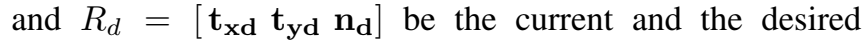
orientation of the master end effector respectively with reference to its base frame. The orientation error $\mathbf{e}_{\mathbf{o}} \in \mathbb{R}^{3}$ between the two configurations is:

$$
\mathbf{e}_{\mathbf{o}}=0.5\left(\mathbf{t}_{\mathbf{x}} \times \mathbf{t}_{\mathbf{x d}}+\mathbf{t}_{\mathbf{y}} \times \mathbf{t}_{\mathbf{y d}}+\mathbf{n} \times \mathbf{n}_{\mathbf{d}}\right)
$$

In order to render the desired torque to the user which matches the control objectives in the four different regions of Fig. 2, the orientation error vector $\mathbf{e}_{\mathbf{o}}$ is scaled by the nonlinear function defined in (1). Hence, the rendered torque $\tau \in \mathbb{R}^{3}$ is equal to:

$$
\boldsymbol{\tau}=f_{\text {scale }} \mathbf{e}_{\mathbf{o}}
$$

\section{Additional Force Profile in Position Regulation}

More properties can be added to the rendered force by combining a second force profile with the normalized nonlinear profile of (1). Our proposed design uses a frictional force model as presented by Bowyer et al. in [7]. The specific model was chosen because it adds motion redirection and dissipates kinetic energy facilitating stability as stated in [7]. However, alternative force models can be used to give different properties to the rendered force. The elasto-plastic frictional force by Bowyer et al. is given by:

$$
\mathbf{f}_{\mathbf{f}}=\sigma_{0} \mathbf{z}_{\mathbf{k}}+\sigma_{1} \dot{\mathbf{z}}_{\mathbf{k}}+\sigma_{2} \dot{\mathbf{p}}
$$

with $\mathbf{z}_{\mathbf{k}} \in \mathbb{R}^{3}$ the elastic displacement and $\mathbf{f}_{\mathbf{f}} \in \mathbb{R}^{3}$ the total frictional force. The first term of (5) represents the frictional force which causes motion redirection (redirects the user back to the surface) while other two are additional damping terms. In our implementation, only the first term of the complete frictional model is used in the conducted experiments presented in Section IV since the friction in the master device damps the motion sufficiently. Hence:

$$
\mathbf{f}=\sigma_{0} \mathbf{z}_{\mathbf{k}}
$$

The elastic displacement $\mathbf{z}_{\mathbf{k}}$ at the $k_{t h}$ time instance is calculated as follows:

$$
\begin{aligned}
& \mathbf{z}_{\mathbf{k}}= \begin{cases}\mathbf{z}_{\mathbf{c}} & \text { if } \Theta\left(\mathbf{z}_{\mathbf{c}}, \mathbf{p}_{\mathbf{t}}\right) \leq \theta \text { and }\left\|\mathbf{z}_{\mathbf{c}}\right\| \leq z_{\mathrm{css}} \\
z_{\mathrm{css}} \frac{\mathbf{z}_{\mathbf{c}}}{\left\|\mathbf{z}_{\mathbf{c}}\right\|} & \text { if } \Theta\left(\mathbf{z}_{\mathbf{c}}, \mathbf{p}_{\mathbf{t}}\right) \leq \theta \text { and }\left\|\mathbf{z}_{\mathbf{c}}\right\|>z_{\mathrm{css}} \\
\hat{\mathbf{z}}_{\mathbf{c}} \hat{\mathbf{y}} & \text { otherwise }\end{cases} \\
& \mathbf{z}_{\mathbf{c}}=\mathbf{z}_{\mathbf{k}-\mathbf{1}}+\left(\mathbf{p}_{\mathbf{k}}-\mathbf{p}_{\mathbf{k}-\mathbf{1}}\right) \\
& \hat{\mathbf{z}}_{\mathbf{c}}= \begin{cases}0 & \text { if } \mathbf{z}_{\mathbf{c}} \hat{\mathbf{y}} \leq 0 \\
\mathbf{z}_{\mathbf{c}} \hat{\mathbf{y}} & \text { if } 0<\mathbf{z}_{\mathbf{c}} \hat{\mathbf{y}}<z_{\mathrm{css}} \\
z_{\mathrm{css}} & \text { otherwise }\end{cases} \\
& \hat{\mathbf{y}}=\operatorname{Rot}(\theta, \hat{\mathbf{m}}) \frac{\mathbf{p}_{\mathbf{t}}}{\left\|\mathbf{p}_{\mathbf{t}}\right\|}, \hat{\mathbf{m}}=\frac{\mathbf{p}_{\mathbf{t}} \times \mathbf{z}_{\mathbf{c}}}{\left\|\mathbf{p}_{\mathbf{t}} \times \mathbf{z}_{\mathbf{c}}\right\|}
\end{aligned}
$$

where $\Theta\left(\mathbf{z}_{\mathbf{c}}, \mathbf{p}_{\mathbf{t}}\right)$ denotes the angle between the candidate elastic displacement $\mathbf{z}_{\mathbf{c}}, \mathbf{p}_{\mathbf{k}} \in \mathbb{R}^{3}$ the current position of the master end effector and the axis $\mathbf{p}_{\mathbf{t}}$ of the conical set $\mathbf{C}_{\mathbf{t}}$ and $\operatorname{Rot}(\theta, \hat{\mathbf{m}})$ the rotation matrix derived from the angle

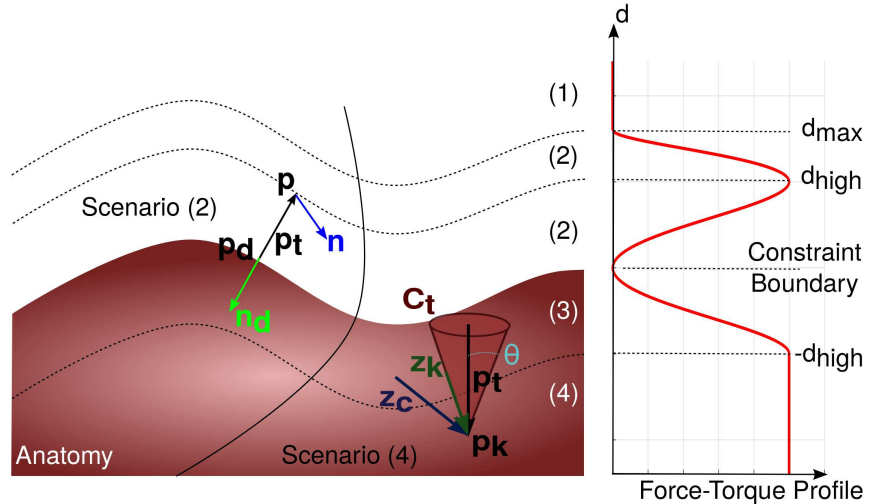

Fig. 2. Zones of proposed controller: Outside of anatomy: (1) Free-hand manipulation and (2) guidance constraints. Inside of Anatomy: (3) guidance and (4) forbidden region active constraints. $\mathbf{p}\left(\mathbf{p}_{\mathbf{k}}\right)$ and $\mathbf{n}$ : current tool pose, $\mathbf{p}_{\mathbf{d}}$ and $\mathbf{n}_{\mathbf{d}}$ : desired tool pose, $\mathbf{p}_{\mathbf{t}}$ : penetration vector, $\mathbf{z}_{\mathbf{k}}$ : current elastic displacement, $\mathbf{z}_{\mathbf{c}}$ : candidate elastic displacement, $\theta$ : apex angle

$\theta$ and the axis $\hat{\mathbf{m}}$ (Fig. 2). The maximum frictional force $f_{c}=\sigma_{0} z_{\mathrm{css}}$ is rendered for distances larger than $z_{\mathrm{css}}$ which is the elastic displacement at steady state with $z_{\mathrm{css}}=d_{\text {high }}$. To achieve force redirection, $\mathbf{z}_{\mathbf{c}}$ is projected onto the conical set $\mathbf{C}_{\mathbf{t}}$ with $\mathbf{p}_{\mathbf{t}}$ the axis of the set and $\theta$ the apex angle (Fig. 2 ). The projection leads to a partial redirection of kinetic energy introduced through motion parallel to the surface. It is worth mentioning that small apex angles result in smaller redirection effects.

The frictional profile prevents free tool manipulation in safe regions. This illustrates the necessity of defining a nonlinear force profile that integrates free tool manipulation with guidance and forbidden region constraints while avoiding switching of the control mode. Hence, the overall force that combines the two control modes is:

$$
\mathbf{F}=f_{\text {scale }} \mathbf{f}
$$

\section{E. Unified Constraints in Position and Orientation}

Using (1)-(4), (6) and (7), the applied joint torques to the left and right master manipulators are equal to

$$
\mathbf{u}=J_{v}^{T} \mathbf{F}+J_{\omega}^{T}\left(\kappa_{o} \boldsymbol{\tau}+\kappa_{\omega} \dot{\mathbf{e}}_{o}\right)+\mathbf{g}(\mathbf{q})
$$

where $J^{T}=\left[\begin{array}{ll}J_{v}^{T} & J_{\omega}^{T}\end{array}\right] \in \mathbb{R}^{k \times 6}$ the Jacobian matrix, $\mathbf{g}(\mathbf{q}) \in$ $\mathbb{R}^{k}$ the vector of gravitational forces, $\mathbf{q} \in \mathbb{R}^{k}$ the vector of the joint angles, $\kappa_{O}$ a positive control constant, $\kappa_{\omega}$ the damping control gain for the angular velocity and $k$ the number of DoF of the master manipulator.

\section{Slave Manipulator Motion Compensation CONTROLLER}

The controller of the slave manipulator accounts for motion following of the target anatomy. The motion compensation scheme proposed in the following considers system delays through online motion prediction. It tracks an arbitrary periodic motion using as input the position of the desired region of the anatomy. This information can be derived from image processing techniques, motion trajectory modelling or tracking of the target anatomy by sensing modalities, such as optical or electromagnetic (EM) trackers. In our 
implementation, EM tracking of the target point is used for demonstration purposes of the algorithm performance; however, the controller functionality is not limited by the input tracking modality. The guided position and orientation of the tool by the master are further superimposed to the motion compensated pose of the slave manipulator creating a semi-static operator frame of reference.

\section{A. Motion Compensation Kinematics}

The translational velocity of the slave manipulator with respect to its base frame ${ }^{b_{s}} \dot{\mathbf{p}}_{s}$ is defined by the velocity attributed to the master manipulator ${ }^{b_{s}} \dot{\mathbf{p}}_{m}$ and the velocity of the surface ${ }^{b_{s}} \dot{\mathbf{p}}_{\text {surf }}$ with respect to the base frame of the slave manipulator $\left\{b_{s}\right\}$. The motion compensated slave velocity ${ }^{b_{s}} \dot{\mathbf{p}}_{s}$ is given by:

$$
\begin{aligned}
{ }^{b_{s}} \dot{\mathbf{p}}_{s} & ={ }^{b_{s}} \dot{\mathbf{p}}_{m}+{ }^{b_{s}} \dot{\mathbf{p}}_{\text {surf }} \\
{ }^{b_{s}} \dot{\mathbf{p}}_{m} & =\alpha^{b_{s}} R_{b_{m}}{ }^{b_{m}} \dot{\mathbf{p}}_{m}
\end{aligned}
$$

where $\alpha$ is the motion scaling factor from the master to the slave workspace, ${ }^{b_{s}} R_{b_{m}}$ the registration matrix between the master slave workspaces and ${ }^{b_{m}} \dot{\mathbf{p}}_{m}$ the velocity of the master with respect to its base frame $\left\{b_{m}\right\}$.

\section{B. Motion Prediction}

To accomplish real-time motion following, it is essential that time delays are considered in the controller design. Online motion prediction is performed in order to address delays introduced by the tracking modality and further system delays. To compensate these delays, motion prediction is required demanding motion modelling. To this end, we perform online signal reconstruction estimating anatomical motion of a tracked point in $\mathbb{R}^{3}$. Hence, the predicted position of the surface ${ }^{b_{s}} \hat{\mathbf{p}}_{\text {surf }}$ is used for motion compensation in the kinematic controller instead of the direct position measurements ${ }^{b_{s}} \mathbf{p}_{\text {surf }}$. Tracking multiple points simultaneously and performing in parallel signal reconstruction for each one of them allows a non-rigid motion compensation. In our experimental implementation, the EM sensor needs to be registered only with the robot base frame since only the relative displacement of the tracked point is necessary to compensate translational motion (9).

The Least-Squares Spectral Analysis (LSSA) is employed for signal reconstruction [15]. LSSA was prefered because it allows unevenly spaced or missing sampling in contrast to the Fourier Transform which demands uniformly spaced samples. Inconsistent sampling can occur when using optical or EM tracking modalities while some feature tracking algorithms have varying processing times depending on the respective image. Interpolation would be necessary to accommodate for this when using FFT to estimate the intermediate samples. The LSSA algorithm requires a number of recorded data points of the trajectory in order to reconstruct the signal. The recorded points $p_{j}$ with $j=1, . ., M$ are used to calculate the dominant frequencies of the signal. The spectral power of the signal is given by:

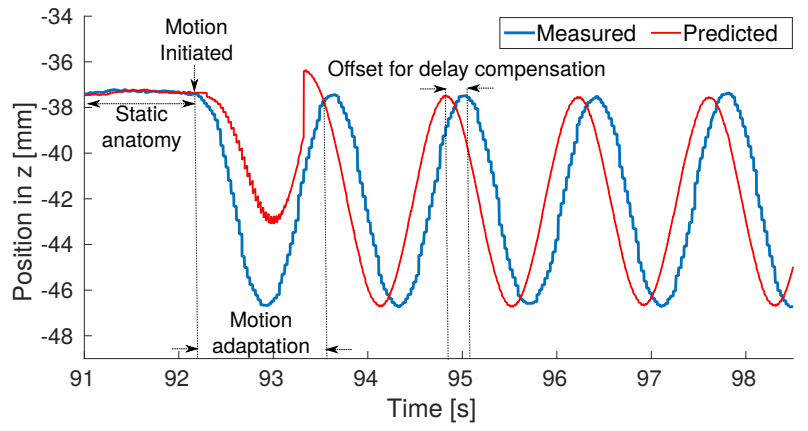

Fig. 3. Trajectory of target as measured by the EM sensor (blue) and predicted trajectory (red) with $A=2 \mathrm{~cm}$ and $T=1.4 \mathrm{~s}$.

$$
\begin{aligned}
P_{N}(\omega)=\frac{1}{2 \sigma^{2}}\left\{\frac{\left[\Sigma_{j}\left(p_{j}-\bar{p}\right) \cos \omega\left(t_{j}-\tau\right)\right]^{2}}{\Sigma_{j} \cos ^{2} \omega\left(t_{j}-\tau\right)}\right. \\
\left.+\frac{\left[\Sigma_{j}\left(p_{j}-\bar{p}\right) \sin \omega\left(t_{j}-\tau\right)\right]^{2}}{\Sigma_{j} \sin ^{2} \omega\left(t_{j}-\tau\right)}\right\}
\end{aligned}
$$

where $\bar{p}, \sigma^{2}$ are the mean value and the variance of the $M$ recorded points respectively, $t_{j}$ the time instance at which $p_{j}$ is acquired and

$$
\tau=\frac{1}{2 \omega} \tan ^{-1}\left(\frac{\Sigma_{j} \sin 2 \omega t_{j}}{\Sigma_{j} \cos 2 \omega t_{j}}\right)
$$

Having obtained the dominant frequencies $\omega_{n}$, the estimation $\hat{p}_{i}(t)$ of $p_{j}$ is reconstructed by linear least-square fitting of the function

$$
\hat{p}_{i}(t)=\alpha_{0}+\sum_{n=1}^{N} A_{n} \sin \left(\omega_{n} t\right)+B_{n} \cos \left(\omega_{n} t\right)
$$

to the one-dimensional signal. In (13), $\hat{p}_{i}(t), i=x, y, z$ denote the coordinates of the predicted position ${ }^{b_{s}} \hat{\mathbf{p}}_{\text {surf }}=\left[\hat{p}_{x}(t) \hat{p}_{y}(t) \hat{p}_{z}(t)\right]^{T}$. Following trajectory reconstruction, the delay offset is added to the signal to compensate system and tracking delays. A measured (blue) and predicted (red) trajectory are depicted in Fig. 3. Note that the reconstructed curve (red) precedes the measured trajectory (blue) to compensate for delays in acquisition.

\section{EXPERIMENTS}

The motor chanelling control scheme described in Sections II and III is implemented on the da Vinci Surgical Platform (Fig. 4) using the da Vinci Research Kit. The proposed framework was integrated into the dVRK using the CISSTSAW libraries and the Robot Operating System (ROS). To demonstrate the effectiveness of the proposed system, two sets of experiments were conducted simulating I) surface scanning for in situ medical imaging and II) vessel manipulation for microvascular anastomosis in Totally Endoscopic Robotic Coronary Artery Bypass. Both tasks were performed by 11 users ( 7 male, 4 female) all of whom were novices. All of the users were able to get familiar with the da Vinci Surgical Platform before performing the experiments. The experiments were performed twice, once with haptic guidance and once in free-hand mode. The parameters of the master active constraints controller are given in Table I. 
TABLE I

PARAMETERS OF THE ACTIVE CONSTRAINTS MASTER CONTROLLER

\begin{tabular}{|c|c|c|c|}
\hline PARAMETER & VALUE & PARAMETER & VALUE \\
\hline$f_{c} \quad[\mathrm{~N}]$ & 5 & $d_{\max }[\mathrm{m}]$ & 0.04 \\
\hline$z_{\mathrm{css}}[\mathrm{m}]$ & 0.02 & $d_{\text {high }}[\mathrm{m}]$ & 0.02 \\
\hline$\sigma_{0} \quad\left[\mathrm{~N} \mathrm{~m}^{-1}\right]$ & 250 & $k_{0} \quad\left[\mathrm{~N} \mathrm{~m}^{-2}\right]$ & $6.7610^{6}$ \\
\hline$[\mathrm{deg}]$ & 5 & {$\left[\mathrm{~m}^{-1}\right]$} & -4 \\
\hline
\end{tabular}

The motion was scaled down by a factor of $\alpha=5$ from the master to the slave workspace. The maximum rendered force $f_{c}$ was set to $5 \mathrm{~N}$ since due to platform constraints it is not feasible to generate hard force feedback to the user. The constraints are activated at $\frac{d_{\max }}{\alpha}=8 \mathrm{~mm}$ from the anatomy in the slave workspace which correspond to $d_{\max }=4 \mathrm{~cm}$ in the master workspace. The maximum force $f_{c}$ is rendered to the user at $\frac{d_{\text {high }}}{\alpha}=4 \mathrm{~mm}$ from the anatomical surface corresponding to $d_{\text {high }}=2 \mathrm{~cm}$ from the virtual constraint boundary. These distances are chosen so that the user can interact with the anatomical surface while preventing further penetration and excessive deformation. In order to get the maximum frictional force $f_{c}$ at distance $z_{\text {css }}=d_{\text {high }}=2$ $\mathrm{cm}$, the parameter $\sigma_{0}$ must be set to $\sigma_{0}=\frac{f_{c}}{d_{\text {high }}}=250$.

\section{A. Surface Scanning for in situ Medical Imaging}

1) Setup and Task: In situ surface scanning requires the operator to maintain a constant contact with a consistent contact force applied to the tissue. It is further important for the image quality to keep the imaging probe orientation parallel to the surface normal. Thereby, tissue deformation should be minimized. Following these requirements, the participants were asked to scan a predefined area of the target anatomy keeping constant contact with the surface and maintaining the tool orientation normal to the surface.

2) Results and Discussion: The objective of the experiment is to demonstrate that the tool motion is regulated around the anatomical surface resulting to reduced deformation and more consistent tool orientation while anatomy penetration is minimized.

The mean values for the free-hand and guided cases along with the corresponding p-values are given in Table II. The p-values were calculated using the Wilcoxon ranksum test since a normal distribution can not be assumed. Maximum penetration and penetration over time are metrics to assess safety improvements while the distance to the surface and mean angle deviation are used as precision metrics. The results presented in Table II show that maximum penetration depth is reduced when active constraints are imposed on the master manipulator in comparison to the free-hand tool manipulation.

The corresponding $p$-value shows that there is a statistically significant difference between the free-hand and the guided case. The mean distance to the surface has a smaller variance and is also significantly reduced when using active constraints (Fig. 8). This demonstrates that the users were able to maintain the tool closer to the anatomy during the scanning procedure in comparison to the free-hand case. Similarly, the mean angle deviation is significantly decreased

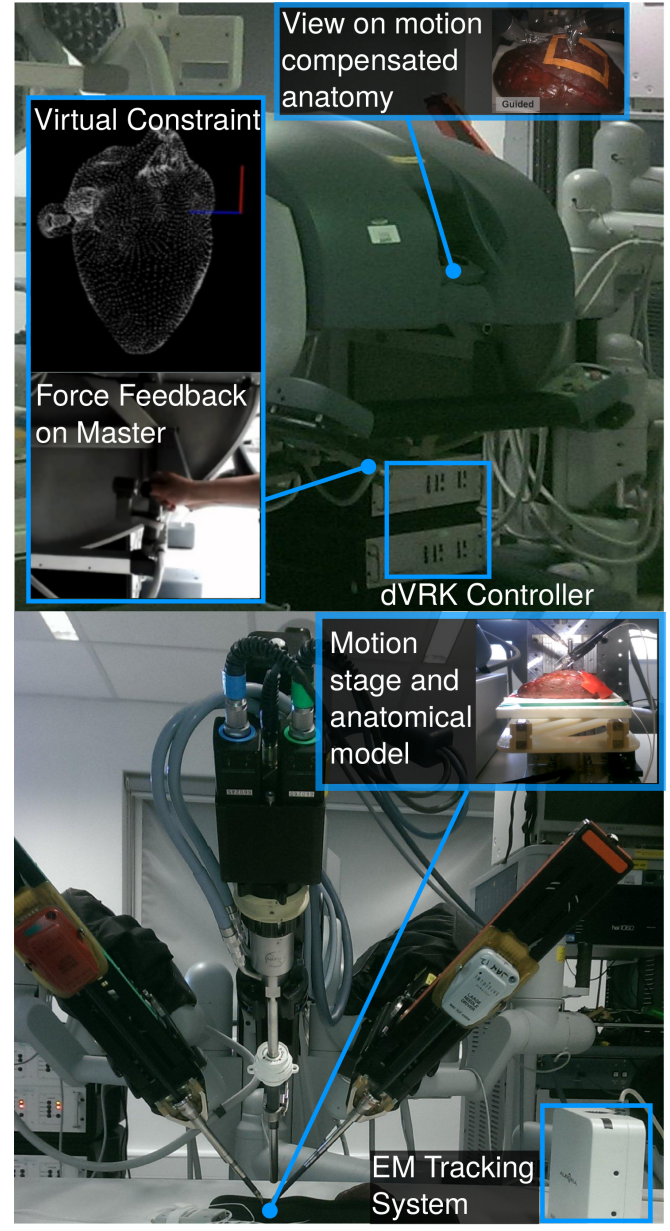

Fig. 4. The da Vinci's Master Console which is used to provide force feedback (top). The slave manipulators of the dVRK used for motion compensation with motion anatomy simulated by a designed stage (bottom)

which indicates that the tool was better aligned with the normal when imposing active constraints (Fig. 5).

An indicative force and tool position dataset as recorded during the user study are depicted in Fig. 9 and 10 respectively. Fig. 9 shows that the proposed profile amplitude is zero when the tool is far outside from the constraint in contrast to the unscaled frictional force which reaches its maximum amplitude at large distances from the constraint irrespectively of anatomy penetration. Hence, the frictional force is turned into a forbidden region profile force despite the fact that it is initially described by the guidance force profile. After the user establishes contact with the anatomy, the distance to surface is regulated to a relatively constant value which is desirable in surface scanning. In Fig. 10, the distance to the anatomy is depicted in case of free (blue) and guided (red) tool manipulation.

It is worth noting that soft tissue allows deformation and hence there is a compromise between safety and precision when working on the constraint boundary. For this reason, soft constraints are imposed on the master allowing the operator to slightly penetrate the constraint boundary. Since low force is experienced around the surface, penetration 
TABLE II

MEAN VALUES AND P-VALUES OF METRICS FOR SURFACE SCANNING

\begin{tabular}{ll|ccc} 
METRIC & & GUIDED & FREE & P-VALUE \\
\hline Mean Max. Penetration & {$[\mathrm{mm}]$} & 3.2 & 6.2 & 0.0001 \\
Penetration Over Time & {$[\mathrm{m} \mathrm{s}]$} & 0.33 & 0.91 & 0.0026 \\
Mean Distance To Surface & {$[\mathrm{mm}]$} & 1.8 & 3 & 0.0001 \\
Mean Angle Deviation & {$[\mathrm{deg}]$} & 27.1 & 59.6 & 0.0003
\end{tabular}

can occur until the retracting force reaches a higher level which warns the user and redirects them back to the surface. The mean maximum penetration ( $3.2 \mathrm{~mm}$ - Table II) occurs slightly before the maximum force is rendered at $4 \mathrm{~mm}$.

The results indicate that the proposed motor chanelling scheme can potentially improve image quality and patient safety. The penetration over time metric also shows reduced values $(\mathrm{p}=0.26 \%)$ when active constraints are imposed on the master manipulator. This demonstrates that during the task, total penetration of the anatomy is significantly minimized using haptic guidance resulting in increased safety and reduced deformation during the performed task.

\section{B. Vessel Manipulation for Microvascular Anastomosis in Coronary Artery Bypass Surgery}

1) Setup and Task: Vessel manipulation in cardiac surgery requires delicate tool operation while the surgeon needs to account for heart motion. The applied tension to the vessel can be minimized by keeping the tool close to the heart surface. This is essential in order to avoid rupturing the vessels due to excessive tension. Moreover, it is significant that the motion of the heart surface is followed facilitating precision and safety.

A rigid phantom was used in order to simulate the target anatomy. The phantom was placed on a platform that was actuated by a linear motor (Faulhaber GmbH \& Co. KG, Germany) in order to simulate cardiac motion along the zaxis. The trajectory of the phantom was tracked by a sensor of an EM tracking system (Aurora, Northern Digital Inc., Canada) attached on the phantom. The tracking system has a measurement rate of $40 \mathrm{~Hz}$. A triangular velocity profile was used for the periodic trajectory with a period of $1.2 \mathrm{~s}$ and $1 \mathrm{~cm}$ amplitude. These values correspond to a motion of 50 beats per minute.

The aim of the second task is to demonstrate how vessel manipulation is performed in a dynamic environment. The participants were asked to grasp the two ends of a vessel and connect them while keeping the tools as close as possible to the heart surface. This vessel manipulation simulates the preparation part of a microvascular anastomosis in which the two ends have to be aligned and connected in order to suture them. The two ends of the vessel were represented by two rubber rings placed on the surface of the phantom.

2) Results and Discussion: The objective of the experiment was to illustrate that precision and safety can be increased when using Active Constraints with motion compensation. The notion is that motion compensation maintains a fixed frame of reference for the user while the Active
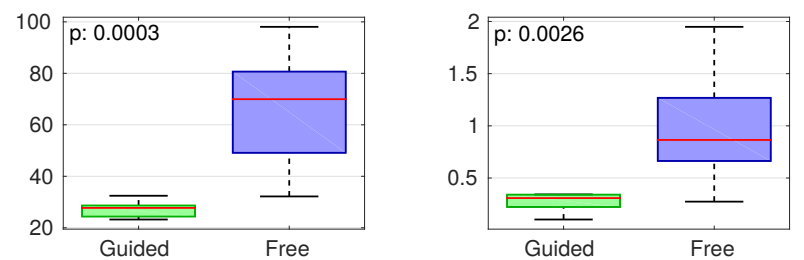

Fig. 5. Mean angle deviation [deg] Fig. 6. Penetration over time [m s]
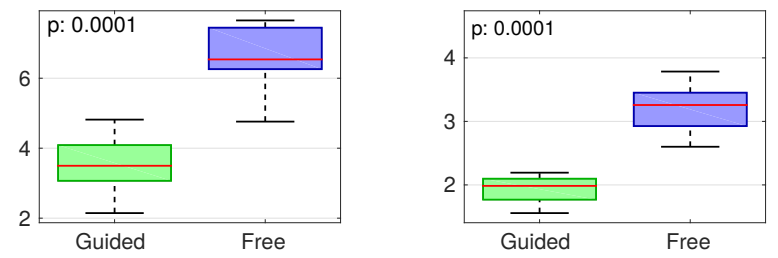

Fig. 7.Mean max. penetration [mm] Fig. 8.Mean distance to surface [mm]

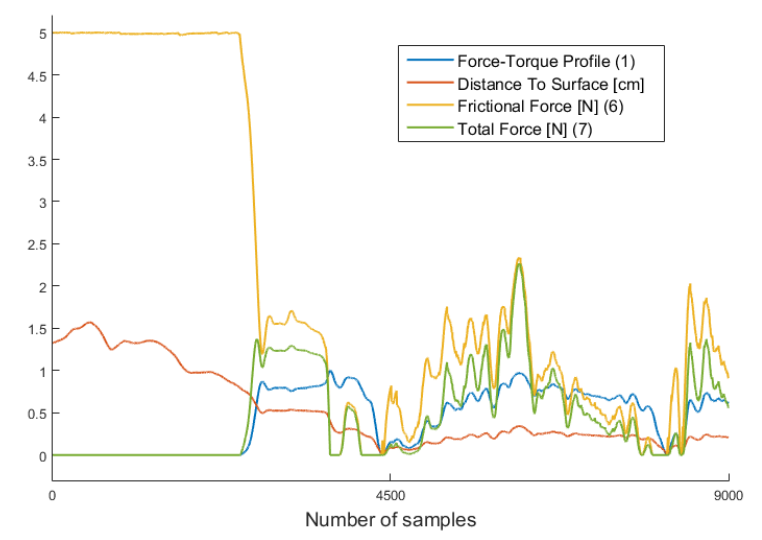

Fig. 9. Constraint forces and tool distance to surface

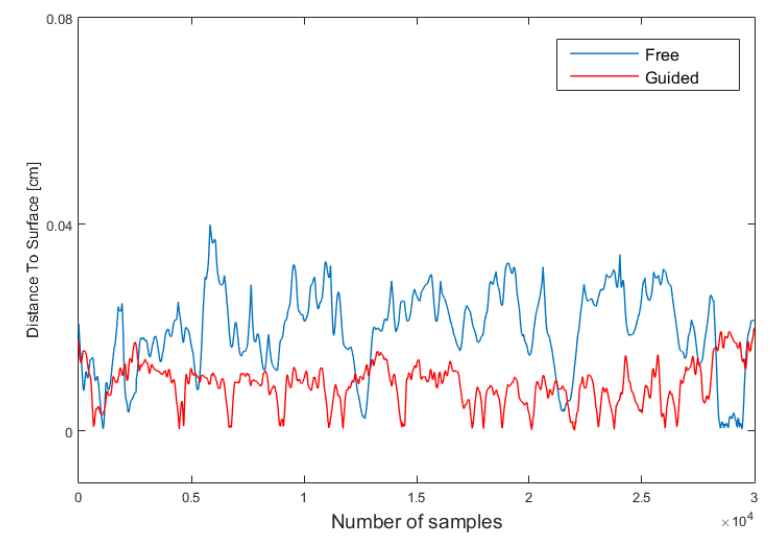

Fig. 10. Distance to anatomy in free and guided mode for surface scanning

Constraints on the master assist the operator in keeping contact with the surface.

Mean distance to the surface, distance over time, mean relative velocity and mean angle deviation are calculated as metrics for this experiment. The corresponding mean values for the free-hand and guided cases along with the corresponding $\mathrm{p}$-values are given in Table III. The p-values 
TABLE III

MEAN VALUES AND P-VALUES OF METRICS FOR VESSEL MANIPULATION

\begin{tabular}{ll|ccc} 
METRIC & & GUIDED & FREE & P-VALUE \\
\hline Mean Relative Velocity & {$[\mathrm{m} / \mathrm{s}]$} & 0.43 & 0.83 & 0.0001 \\
Distance Over Time & {$[\mathrm{m} \mathrm{s}]$} & 0.56 & 1.102 & 0.026 \\
Mean Angle Deviation & {$[\mathrm{deg}]$} & 22.35 & 47.4 & 0.0001
\end{tabular}
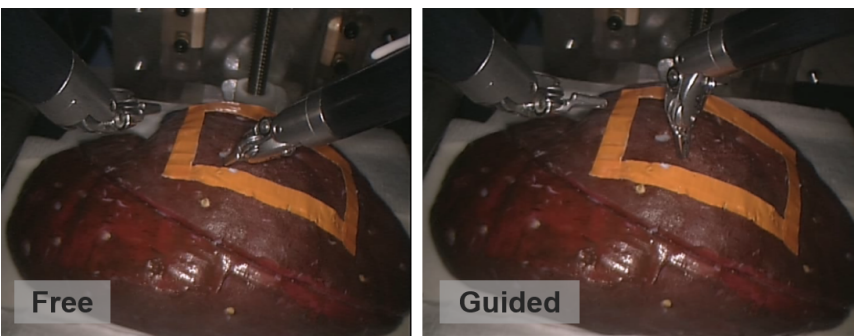

Fig. 11. Tool orientation during surface scanning without (left) and with (right) guidance as viewed through the endoscopic camera

given in the third column of Table III were again calculated using the Wilcoxon ranksum test. The metrics presented in Table III show that the mean relative velocity between the tool and the surface is significantly decreased when motion is compensated by the slave manipulator. The distance covered by the tool over time, i.e. the integral of the distance to the surface with respect to time, is also minimized when motion compensation and haptic guidance are imposed (Fig. 12). This illustrates that constant tool movement compensating heart motion reduces the distance traveled relative to the anatomy. It also shows more consistent tool manipulation with close proximity to the anatomy. The p-value of this metric states statistically significant difference between the two cases. Furthermore, the mean angle deviation is decreased similarly to the first user study (Fig. 13).

\section{CONCLUSIONS}

In this paper, a motor chanelling control strategy for bimanual tasks comprising Active Constraints and motion compensation is presented. Guidance and Forbidden Region Constraints are generated by a nonlinear profile that integrates both types of constraints in a unified control law. The dynamics of the surgical environment are considered in the design by compensating motion of the anatomy with online modelling. Motion prediction in $3 \mathrm{DoF}$ is performed in order to accomplish real-time compensation and overcome system and tracking delays. The proposed framework is implemented on the da Vinci Surgical Platform using the dVRK. The experimental results of the user study indicate that the presented control approach outperforms free-hand manipulation improving safety and precision in the simulated surgical scenarios.

\section{ACKNOWLEDGMENTS}

The authors would like to thank Lin Zhang for his input on the dVRK, Petros Giataganas and Piyamate Wisanuvej for their contribution to the hardware components in this work and Dr. Philip Pratt for the endoscopic camera software interface.
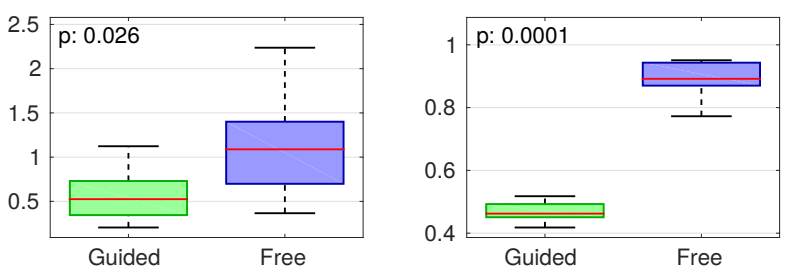

Fig. 12. Distance Over Time [m s] Fig. 13. Mean Relative Velocity [m/s]

\section{REFERENCES}

[1] S. Park, R. D. Howe, and D. F. Torchiana, "Virtual fixtures for robotic cardiac surgery," in International Conference on Medical Image Computing and Computer-Assisted Intervention. Springer, 2001, pp. 1419-1420.

[2] J. Ren, R. V. Patel, K. A. McIsaac, G. Guiraudon, and T. M. Peters, "Dynamic 3-D virtual fixtures for minimally invasive beating heart procedures," IEEE Transactions on Medical Imaging, vol. 27, no. 8, pp. 1061-1070, 2008.

[3] A. Kapoor and R. H. Taylor, "A Constrained Optimization Approach to Virtual Fixtures for Multi-Handed Tasks," in IEEE International Conference on Robotics and Automation, 2008, pp. 639-644.

[4] P.-L. Yen and B. L. Davies, "Active constraint control for imageguided robotic surgery." Proceedings of the Institution of Mechanical Engineers. Part H, Journal of engineering in medicine, vol. 224, pp. 623-631, 2010.

[5] F. Ryden and H. J. Chizeck, "Forbidden-region virtual fixtures from streaming point clouds: Remotely touching and protecting a beating heart," in IEEE International Conference on Intelligent Robots and Systems, 2012, pp. 3308-3313.

[6] M. Li, M. Ishii, and R. H. Taylor, "Spatial Motion Constraints Using Virtual Fixtures Generated by Anatomy," vol. 23, no. 1, pp. 4-19, 2007

[7] S. A. Bowyer and F. Rodriguez Y Baena, "Dynamic Frictional Constraints in Translation and Rotation," in 2014 IEEE International Conference on Robotics and Automation, 2014, pp. 2685-2692.

[8] T. L. Gibo, L. N. Verner, D. D. Yuh, and A. M. Okamura, "Design considerations and human-machine performance of moving virtual fixtures," 2009 IEEE International Conference on Robotics and Automation, pp. 671-676, 2009.

[9] K. Leibrandt, H. J. Marcus, K. W. Kwok, and G. Z. Yang, "Implicit Active Constraints for a Compliant Surgical Manipulator," in 2014 IEEE International Conference on Robotics and Automation, 2014, pp. 276-283.

[10] K. W. Kwok, K. Hung Tsoi, V. Vitiello, J. Clark, G. C. T. Chow, W. Luk, and G. Z. Yang, "Dimensionality reduction in controlling articulated snake robot for endoscopy under dynamic active constraints," IEEE Transactions on Robotics, vol. 29, pp. 15-31, 2013.

[11] A. Ruszkowski, O. Mohareri, S. Lichtenstein, R. Cook, and S. Salcudean, "On the feasibility of heart motion compensation on the davinci $\mathbb{R}$ surgical robot for coronary artery bypass surgery: Implementation and user studies," in 2015 IEEE International Conference on Robotics and Automation (ICRA), 2015, pp. 4432-4439.

[12] S. G. Yuen, D. T. Kettler, and R. D. Howe, "Robotic motion compensation for beating intracardiac surgery," 2008 10th International Conference on Control, Automation, Robotics and Vision, ICARCV 2008, vol. 28, no. 10, pp. 617-622, 2008.

[13] P. Kazanzides, Z. Chen, A. Deguet, G. S. Fischer, R. H. Taylor, and S. P. DiMaio, "An open-source research kit for the da Vinci® Surgical System," in 2014 IEEE International Conference on Robotics and Automation (ICRA), 2014, pp. 6434-6439.

[14] K. Leibrandt, C. Bergeles, and G.-Z. Yang, "On-Line Collision-Free Inverse Kinematics with Frictional Active Constraints for Effective Control of Unstable Concentric Tube Robots," in Intelligent Robots and Systems (IROS), 2015 IEEE/RSJ International Conference on, 2015.

[15] N. R. Lomb, "Least-squares frequency analysis of unequally spaced data," Astrophysics and space science, vol. 39, no. 2, pp. 447-462, 1976. 